Supporting Information for

\title{
Phase Behavior of Salt-Doped A/B/AB Ternary Polymer Blends: The Role of Homopolymer Distribution
}

\author{
Shuyi Xie, ${ }^{1}$ Bo Zhang, ${ }^{2}$ Frank S. Bates, ${ }^{2}$ Timothy P. Lodge ${ }^{* 1,2}$ \\ ${ }^{1}$ Department of Chemistry and ${ }^{2}$ Department of Chemical Engineering and Materials \\ Science, University of Minnesota, Minneapolis, MN 55455
}


(a)

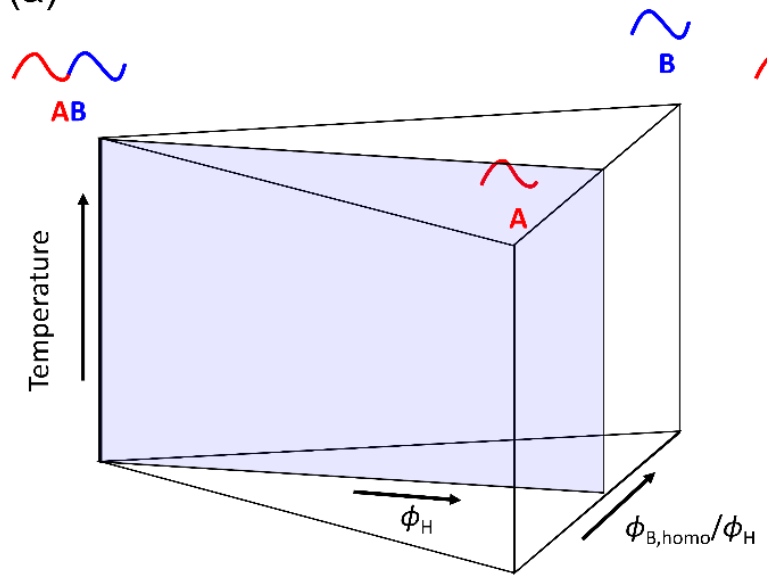

(b)

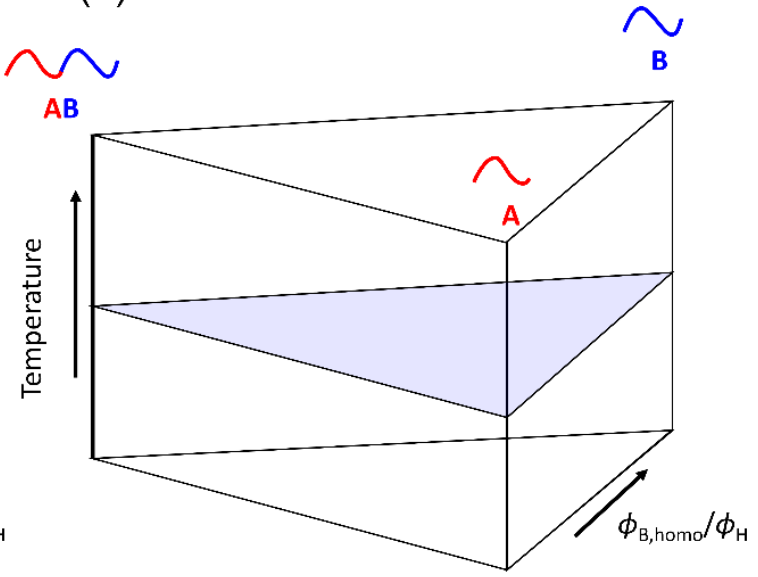

Figure S1. Schematic of (a) an isopleth and (b) an isothermal slice in the $\mathrm{A} / \mathrm{B} / \mathrm{AB} 3 \mathrm{D}$ phase prism.

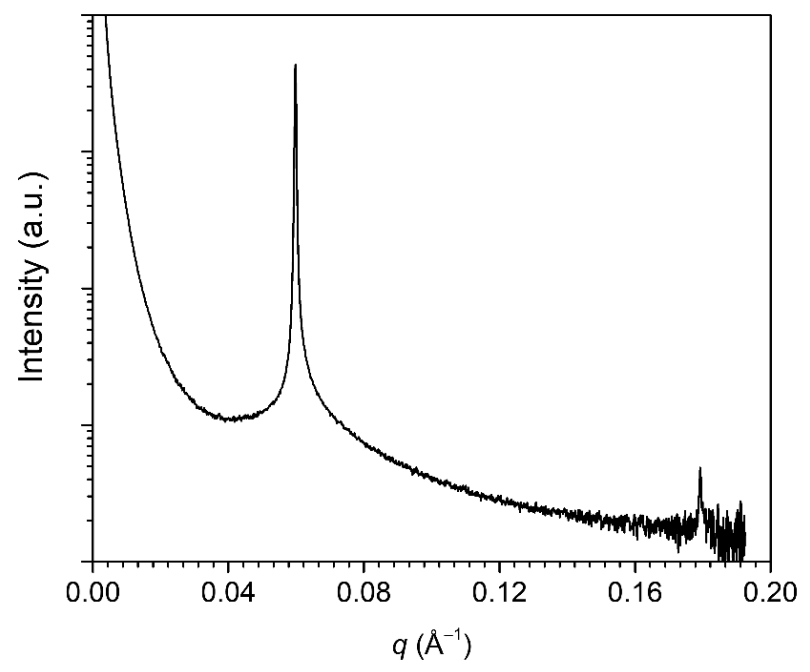

Figure S2. SAXS profile of SO with $r=0.06$ at $120^{\circ} \mathrm{C}$. The secondary peak for LAM is missing due to form factor extinction. 


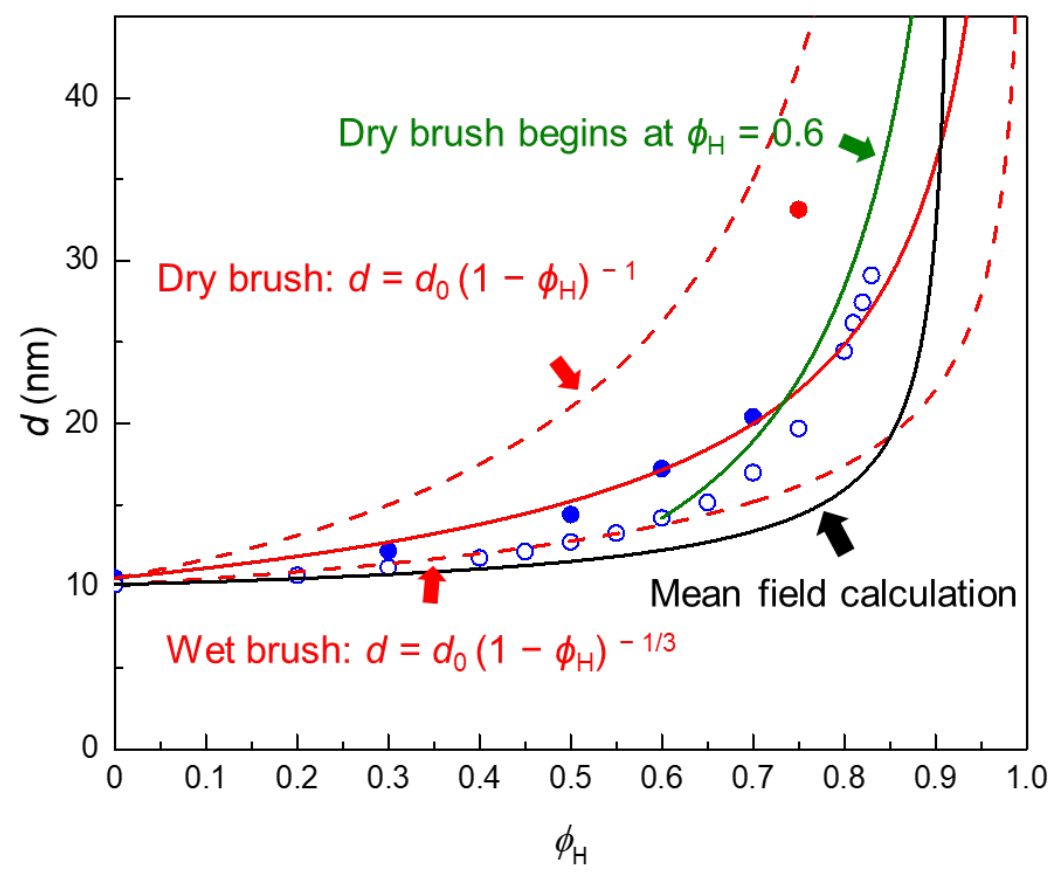

Figure S3. Characteristic size (the center of one A domain to the next) for blends with $r$ $=0.03$ (open symbols) and $r=0.06$ (solid symbols). This figure refers to Figure 3 . The green curve is the prediction of the dry brush behavior starting at $\phi_{\mathrm{H}}=0.6$ for blends with $r=0.03$. 
(a)

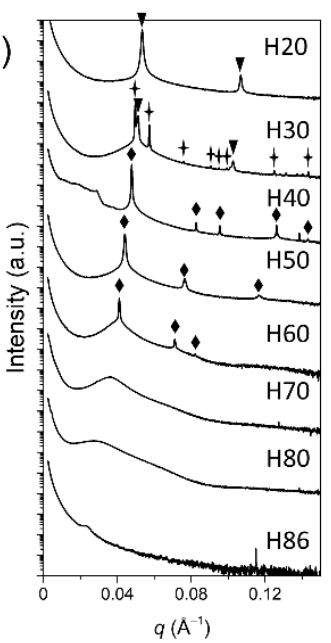

(e)

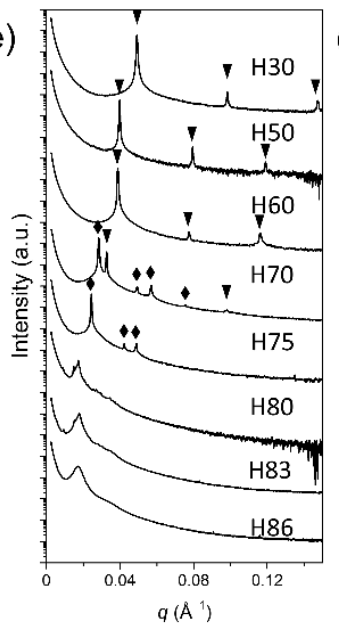

b)

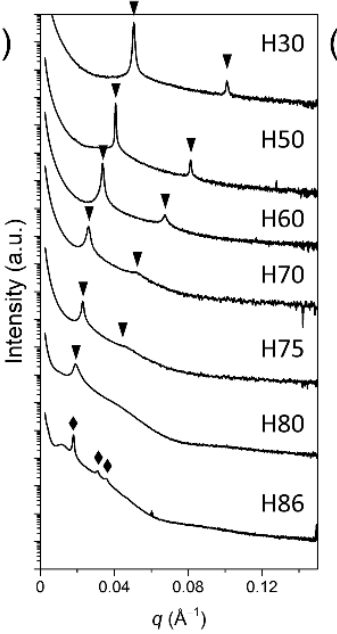

(f)

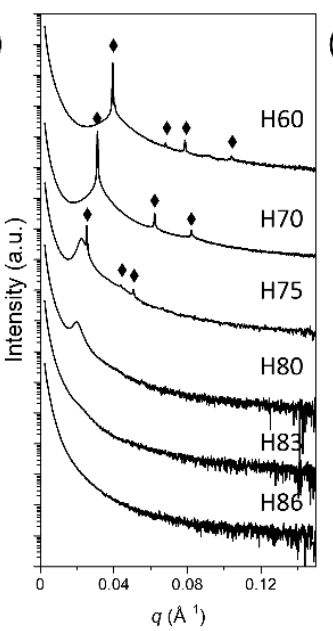

(c)

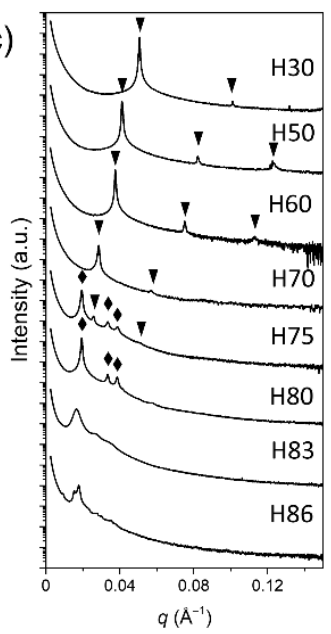

(g)

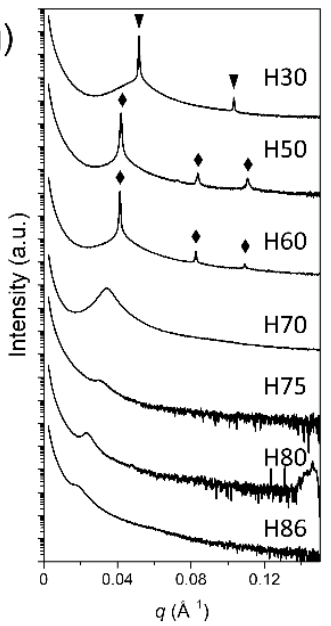

(d)

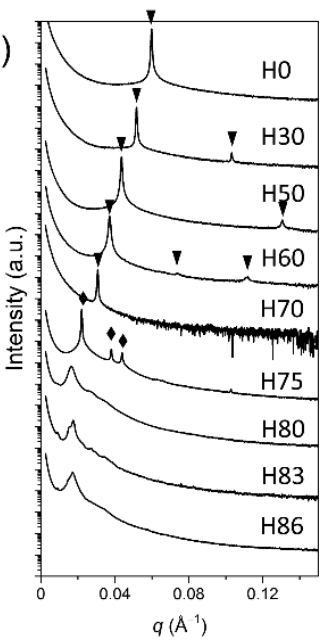

(h)

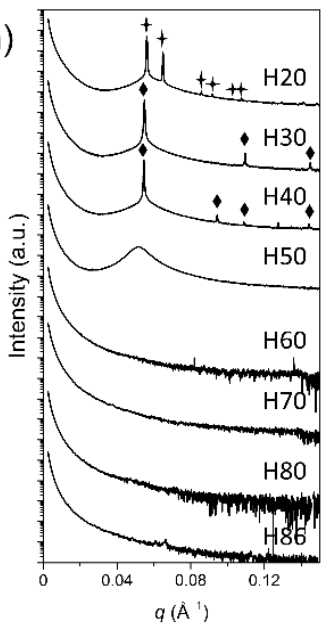

Figure S4. SAXS patterns for LiTFSI-doped S/O/SO blends with $r=0.06$ and at $120{ }^{\circ} \mathrm{C}$ along the (a) EO0, (b) EO20, (c) EO40, (d) EO50, (e) EO60, (f) EO70, (g) EO80, and (h) EO100 isopleths, and with different $\phi_{\mathrm{H}}$. The relative peak positions of LAM at $q / q^{*}$ $=1,2,3 \ldots$ are marked by inverse triangles; HEX phase at $q / q^{*}=1, \sqrt{3}, \sqrt{4}, \sqrt{7}, \sqrt{9}$ ... are marked by diamonds, where $q^{*}$ is the primary peak; GYR phase at relative $q$ positions of $\sqrt{6}, \sqrt{8}, \sqrt{14}, \sqrt{16}, \sqrt{20}, \sqrt{22} \ldots$ are marked by four-pointed stars. 

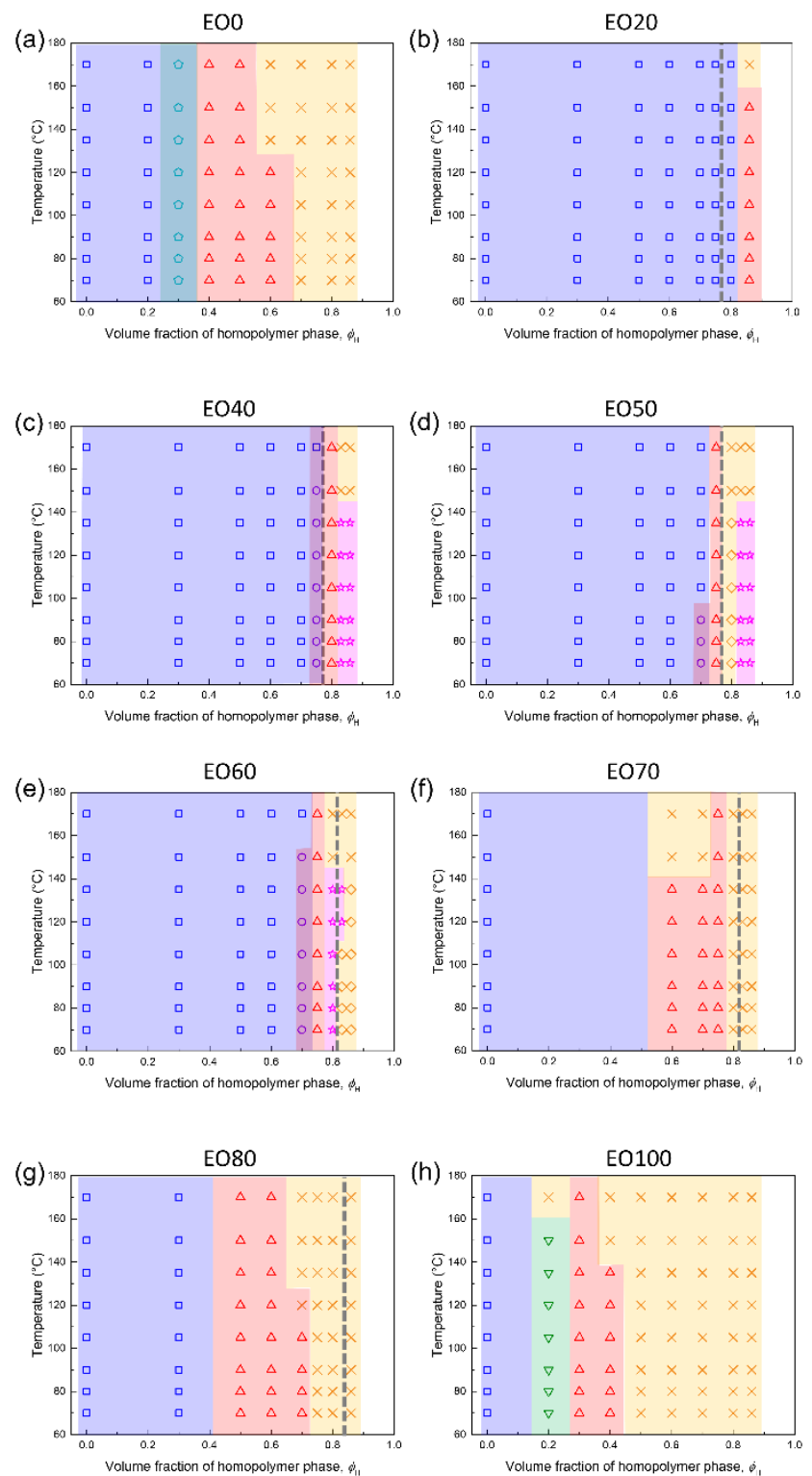

Figure S5. Phase diagrams along the isopleths with (a) EO0, (b) EO20, (c) EO40, (d) EO50, (e) EO60, (f) EO70, (g) EO80, and (h) EO100. The shapes of the symbols correspond to the morphologies of the blends: $\square$ for LAM (blue), $\nabla$ for GYR (green), $\Delta$ for HEX (red), is for C15 (magenta), $\diamond$ for LLP (yellow), $X$ for DIS (yellow), $\diamond$ for LAM/GYR and $\circ$ for LAM/HEX. The shaded regions represent phases with specific morphologies indicated by the color, and phase boundaries are estimated based on compositions for different morphologies. The dashed vertical lines indicate the miscibility limit above which macroscopic phase separation occurs. 


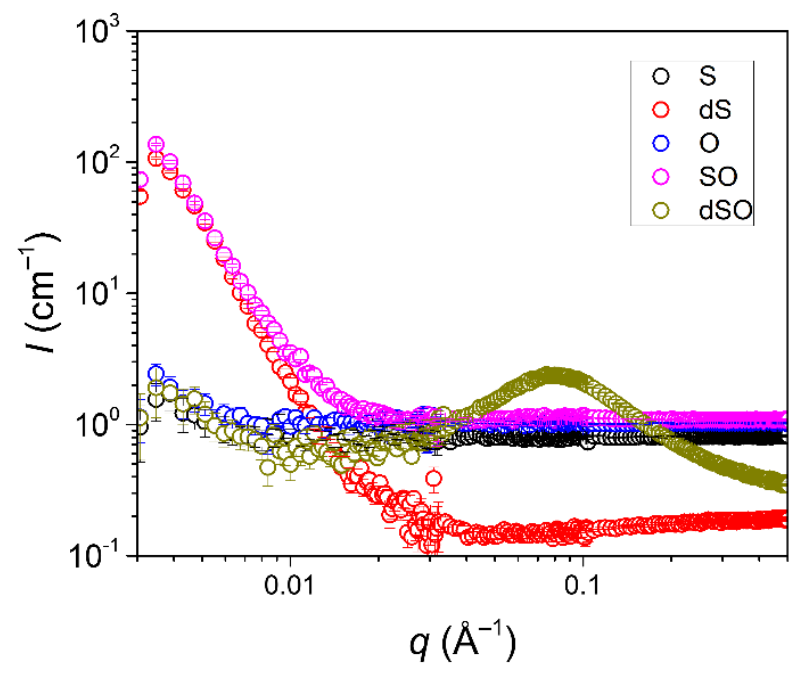

Figure S6. Incoherent SANS intensity in absolute units for polystyrene (S), deuterated polystyrene (dS), poly(ethylene oxide) (O), PS- $b$-PEO (SO), and dPS- $b$-PEO (dSO) acquired at $120^{\circ} \mathrm{C}$. 

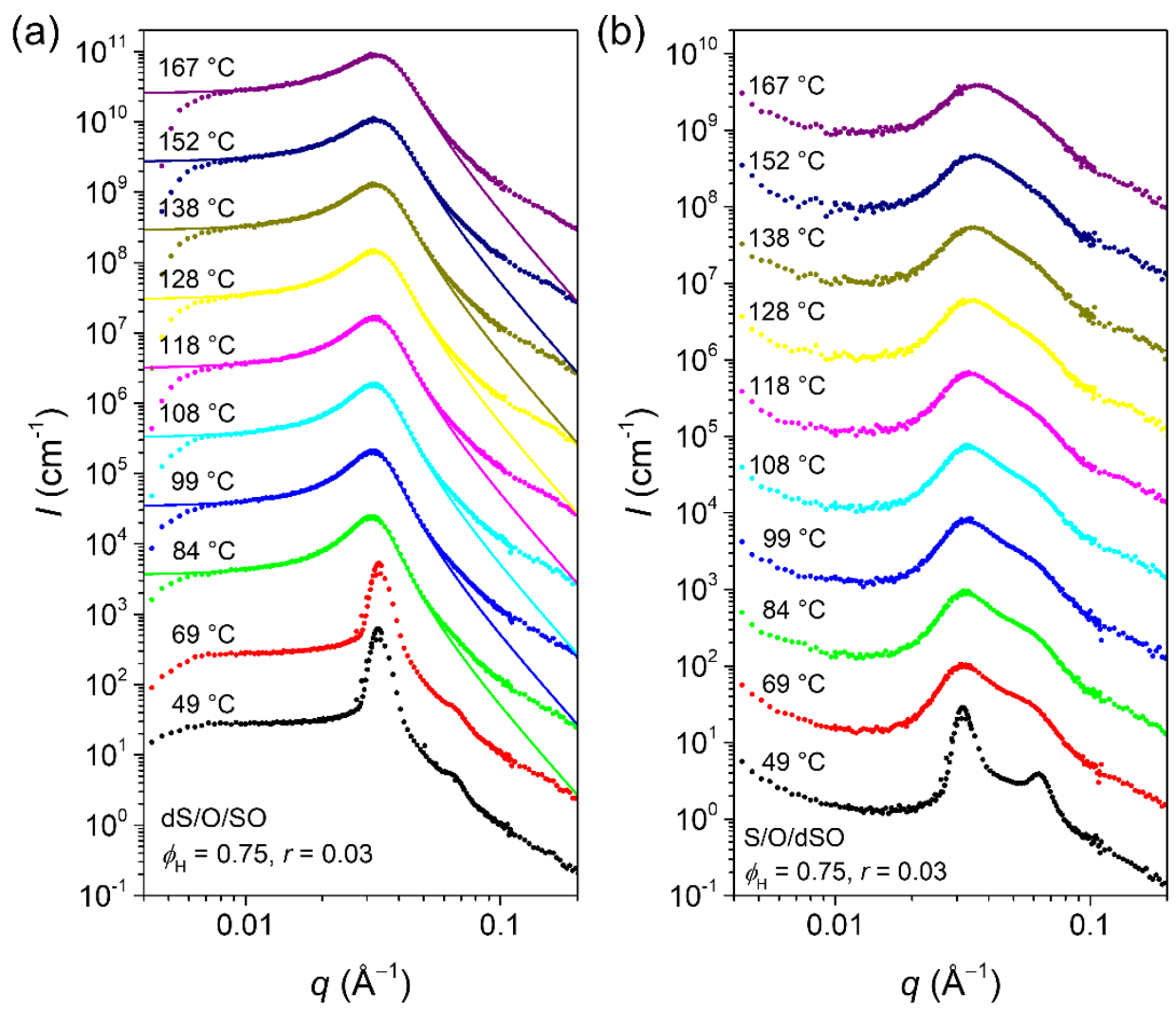

Figure S7. SANS intensity in absolute units acquired as a function of temperature for the LAM structure with different labeling: (a) $\mathrm{dS} / \mathrm{O} / \mathrm{SO}$ and (b) $\mathrm{S} / \mathrm{O} / \mathrm{dSO}$ with $r=0.03$ and $\phi_{\mathrm{H}}=0.75$. The solids curves are the optimized T-S fits. All the scattering patterns have been vertically shifted successively by factors of 10 . 